\title{
La relación educación-sociedad en el discurso político- educativo de los gobiernos de la Concertación (1990-2009)*
}

The relationship between education and society within the political-educational discourse of Gobernments of Concertación (1990-2009)

Relação educação-sociedade no discurso político e educacional dos governos de coalizão (1990-2009)

\section{Juan E. García-Huidobro, ${ }^{a}$ Rocío Ferrada, ${ }^{b}$ Marcela Gil ${ }^{c}$}

aFacultad de Educación, U. Alberto Hurtado. Telf.: (562) 28897100. Correo electrónico: jgarciah@uahurtado.cl

bDoctorante GIRSEF-U.C.L., Bélgica. Correo electrónico: rferrada@uahurtado.cl

cCIDE, U.A.H.. Correo electrónico: mgil@cide.cl

\section{RESUMEN}

Este artículo examina la visión de sociedad que acompaña a la política educativa de los gobiernos de la Concertación. Para ello estudia los 'discursos de inauguración del año escolar' pronunciados por los ministros de educación entre 1990-2009. En ellos se consigna los conceptos de sociedad utilizados expresamente y se examina también aquellas concepciones a las que se recurre, de modo indirecto, para justificar las medidas de política educativa propuestas. Se organiza los discursos en tres períodos. En el primer momento se marca con claridad la identidad del nuevo gobierno y las diferencias con la sociedad heredada de la dictadura. En la segunda etapa predomina la presentación y justificación de las reformas y realizaciones. En el período de cierre se enfrenta demandas educativas fuertes y el discurso rencuentra los énfasis democráticos e igualitarios del discurso inicial.

Palabras clave: Gobiernos de la Concertación, 1990-2009, visión de sociedad, discurso político, discurso educativo.

\begin{abstract}
This article examines the vision of society that goes together with the educational policies of the governments of the Concertación. In order to do this, the author studies the 'inauguration speeches of the school year' given by the Ministers of Education between 1990 and 2009. In them, the concepts of society expressly used are reviewed and so are those conceptions, indirectly used, to justify the measures of educational policies proposed. The educational political speech of the Concertación is analyzed between 1990 and 2009, organizing it into three periods. At first, the identity of the new government and the differences with the inherited society from the dictatorship are clearly marked. In the second stage, the presentation and justification of the reforms and accomplishments are predominant. The closure period is faced with strong education demands and the speech encounters again the democratic and egalitarian emphasis of the original speech.
\end{abstract}

Key words: Coalition governments of Chile (Concertación), 1990-2009, vision de society, political discourse, educational discourse.

\section{RESUMO}

Analisa-se a visão de sociedade que acompanha a política educacional dos governos de Coalizão. Para isto, estudamse os 'discursos de abertura do ano escolar' proferidos pelos Ministros da Educação de 1990 a 2009. Deles, retira-se o conceito de sociedade e neles também se examina concepções às quais se recorre, de modo indireto, para justificar

Investigación realizada en el marco de CEPPE (Centro de Estudios de Prácticas y Políticas en Educación), con el auspicio de CONICYT (Comisión Nacional de Investigación Científica y Tecnológica). Los autores pertenecen a la Facultad de Educación de la Universidad Alberto Hurtado. 
as medidas políticas propostas para a educação. Analisa-se o discurso político educativo da Coalizão entre os anos de 1990 a 2009, organizando-o em três períodos. No primeiro, está expressa claramente a identidade do novo governo e as diferenças com a sociedade do período da Ditadura. No segundo, predomina a apresentação e justificativas de reformas e realizações. No último, enfrenta-se demandas educativas fortes e o discurso enfatiza a visão democrática e igualitária do discurso inicial.

Palavras chave: Governos de Coalizão do Chile (Concertación), 1990-2009, visão de sociedade, discurso político, discurso educativo.

\section{INTRODUCCIÓN}

Este artículo estudia la visión de sociedad en la que se sustenta y a la que buscó contribuir la política educativa de los gobiernos de la Concertación. Subyace a este propósito, el supuesto de que las políticas educativas no poseen finalidades autónomas, sino que reciben su orientación del tipo de sociedad que se desea; la que a su vez se conecta con una determinada visión ético política respecto a cómo debe ser el orden social.

Para cumplir este objetivo se examina principalmente los discursos de inauguración del año escolar pronunciados por los ministros de educación entre 1990-2009. En ellos se inspecciona los conceptos de sociedad utilizados expresamente y se examina también aquellos conceptos a los que se recurre de modo indirecto, para justificar el tipo de educación o las medidas de política educativa propuestas.

Esta mirada retrospectiva parece particularmente relevante en un momento en el cual, motivados por el movimiento y las demandas estudiantiles del 2011-2012, existe una crítica bastante extendida, que propone superar el carácter mercantil del modelo de organización que ha primado en la educación chilena los últimos treinta años.

La primera parte del texto explicita el marco analítico y describe los documentos analizados. En la segunda parte, se examina el discurso político educativo de la Concertación, entre los años 1990 a 2009, organizado en tres períodos. Para finalizar, se subraya algunas conclusiones.

\section{ANTECEDENTES DEL ANÁLISIS}

\subsection{RELACIÓN EDUCACIÓN Y SOCIEDAD: FUNCIONES DE LA EDUCACIÓN}

Desde un punto de vista social, la institución educativa se ha pensado -y diseñadocomo funcional a un determinado proyecto de sociedad. Esta relación supone que la educación, en cuanto institución social, adquiere sentido en referencia a un determinado ideal de sociedad o propuesta sobre el orden que queremos. ${ }^{1}$ Tal lógica pone de manifiesto el carácter político de toda educación, puesto que ella debe responder a la pregunta por los fines de la sociedad deseada. También pone en evidencia el referente trascendente que la determina y le da su razón de ser, ya que la educación debe tener en cuenta cuestiones tales como: ¿qué tipo de ser humano se quiere formar? ¿Qué ideal de relaciones se propician entre las personas y de éstas con el Estado y la colectividad? ¿Qué criterios se

La vinculación teórica entre la idea de sociedad y el tipo de educación ha sido consignada desde antiguo (en Platón y Aristóteles) y se presenta particularmente en el proyecto republicano, cuyo ideal de gobierno corresponde a un régimen de homogeneidad entre las instituciones del Estado y las costumbres de la sociedad. Ver Guttman (2001). 
quiere promover para distribuir lo común? Por el contrario, una educación no vinculada con estas finalidades se vacía de su sentido social y deviene irreflexiva e intrascendente. ${ }^{2}$

En las actuales sociedades, modernas y globalizadas, es posible distinguir al menos tres funciones que la educación debe cumplir en su tarea de formar a las personas con las aptitudes y capacidades necesarias para desempeñarse en ellas.

a) Una primera corresponde a su aporte al desarrollo personal. La educación es un proceso que busca que el estudiante desenvuelva las capacidades necesarias para comprender el mundo y para estar en mejor posición de dar sentido a su vida.

Esta dimensión ha sido consagrada en la Declaración de los Derechos Humanos y en la Carta Constitucional de nuestro país. Más recientemente, el Informe Delors: La educación encierra un tesoro (UNESCO, 1997), define como uno de los pilares de la educación para el siglo XXI, el "aprender a ser", invocando su capacidad para apoyar el despliegue de la propia personalidad, de forma que los sujetos estén en condiciones de obrar con creciente autonomía y responsabilidad personal.

b) La segunda es la función sociopolítica de la educación, por la cual es responsable de formar los valores políticos, las actitudes y las formas de comportamiento de los futuros ciudadanos, cuya virtud distintiva deberá ser su capacidad para influir en la forma en que se produce y reproduce la sociedad. "Esto significa que esa sociedad debe educar a todos los niños para que sean capaces de participar en la definición colectiva de la sociedad" (Guttman, 2001: 60).

La capacidad de la institución educativa para formar a la población en un conjunto de valores, normas sociales y hábitos compartidos que refuerzan el sentimiento de pertenencia a una comunidad, haciendo más probable la concertación de ideas, fue destacada por Durkheim (1977 y 1998). El problema de la integración social, de la vida en comunidad, de la cohesión social entre individuos distintos, sólo es posible, según el autor, por la existencia de un sustrato moral que sostiene las relaciones sociales -y no por el contrato, como se piensa desde una perspectiva individualista (por ejemplo, ver Tironi, 2007).

Desde un enfoque republicano, la escuela y la universidad son también espacios públicos abiertos a la deliberación y al diálogo, al encuentro con formas de vida diferentes, donde se comparte una igualdad básica, tornando a la diversidad social algo posible y deseable, en tanto capaz de un ejercicio dialógico orientado al consenso (Peña, 2008).

En su sentido sustantivo, la democracia presupone la igualdad. Invocar este principio permite debatir, conformar y reclamar la distribución del orden dado a fin de comprobar la pertenencia a una comunidad política en igual calidad (Rancière, 2006). Esta comunidad es el producto de un acuerdo entre sujetos políticos alcanzado mediante el diálogo en el espacio público; la concurrencia al diálogo en condición de igualdad es la acción propiamente ciudadana. ${ }^{3}$

En este punto es imposible no subrayar la coyuntura histórica. Con el inicio de los gobiernos concertacionistas en 1990, no solo retorna un régimen político democrático, sino

2 Esta pérdida de sentido o "declive de las instituciones" por falta de referentes que apelen a la idea de unidad social, de un todo homogéneo y coherente, es un fenómeno característico de las actuales sociedades modernas. Ver Dubet (2002 y 2007). Sobre los efectos de este proceso en el campo educativo ver Tedesco (1995 y 1998).

3 La razón dialógica, cuyo elemento es el discurso o la palabra, es lo propio de la esfera política. La política propiamente dicha empieza con el diálogo y la instauración de las libertades, del derecho a tener derechos, reconocidos en el espacio público (Habermas, 1981; Arendt, 2005). 
que se busca reponer "la democracia" como orden sociopolítico y horizonte normativo de la acción del Estado. Esto es, se asume una posición ético-política democrática, como principio y fin de la acción estatal y como fundamento de nuestra organización social y política. Ahora bien, dado que a ser ciudadano se aprende (Cortina, 1997), sería del todo esperable una presencia importante de reflexiones y acciones educativas que refieran a objetivos de formación ciudadana y cohesión social y, por ende, a la importancia de formar individuos capaces de dialogar, de interesarse por los asuntos comunes, de juzgar sus relaciones con otros y con la colectividad.

c) La tercera es la función económica de la educación, ya destacada en la teoría del "capital humano" (Becker, 1983) como factor productivo fundamental para el desarrollo económico de los países, por cuanto una fuerza laboral más educada generaría mayor productividad. Esta relación es hoy fundamental en la nueva economía global basada en el conocimiento, al punto que en la economía informacional, la educación y la innovación se constituyen en fuerzas productivas directas (Castells, 2005). ${ }^{4}$

En relación a esta tercera función hay una convergencia histórica a destacar. En 1990, en el mismo momento en que Chile iniciaba su vuelta a la democracia y prometía "desarrollo con equidad" (Castells, 1995), la CEPAL señalaba que la transformación productiva con equidad era la tarea prioritaria del desarrollo de América Latina y el Caribe en los años noventa (CEPAL, 1990).

En 1992 CEPAL, en asociación con la oficina regional de UNESCO en Santiago, definió la educación y el conocimiento como eje de su propuesta de transformación productiva con equidad. Esta propuesta, que -como veremos- fue cercana al discurso educacional de la Concertación, tiene el mérito de sintetizar bien las distintas funciones que se le han dado a la educación. Retoma la función de integración social que, en las sociedades modernas, le ha sido asignada a la educación -por cierto, especialmente a la educación pública. Incluye su función sociopolítica y económica, y señala que ella es crucial para enfrentar el desafío de la formación ética y ciudadana, y de una convivencia propiamente democrática (función política), así como para la generación de capacidades y destrezas indispensables para la competitividad económica internacional, crecientemente basada en el progreso técnico (función económica).

Las funciones sociales de la educación y las exigencias que hace a la educación la adhesión a un horizonte normativo democrático, que acabamos de revisar, son los elementos que distinguiremos a continuación en los discursos de política educativa de los gobiernos de la Concertación, durante los años 1990 a 2009. A partir de este análisis, intentaremos caracterizar la relación entre educación e idea del orden social que se hace presente en ellos. Se trata de una tarea necesaria, ya que los discursos acerca de la sociedad -donde, por cierto, los discursos político-educativos no son una excepción- no sólo describen y legitiman la realidad que enuncian, sino que en este mismo acto, también la modifican, en la medida que marcan los límites de lo posible, orientan la acción política y favorecen opciones ligadas a intereses determinados.

$4 \quad$ No obstante, siendo condiciones necesarias no son suficientes para alcanzar el desarrollo. Castells explica que: "en la medida que el excedente se transfiere al mercado financiero, el comportamiento de éste sometido a percepciones de sicología colectiva y turbulencias informativas de todo origen, influye decisivamente en la riqueza y pobreza de las naciones" (Castells, 2005: 19). 


\subsection{DOCUMENTOS ESTUDIADOS}

Para estudiar la relación indicada, acudimos a dos fuentes representativas del discurso político educativo de la Concertación. Estas son: i) Los discursos de inauguración del año escolar, 1990-2009. Recurrentemente en estos discursos el ministro(a) de la cartera informa acerca de la política educativa implementada y suele anunciar y justificar las propuestas educativas a realizar durante el año escolar que abre. ii) Los mensajes del ejecutivo, que encabezan los proyectos de ley sobre educación ingresados al Parlamento durante los cuatro gobiernos de la Concertación. Estos mensajes contienen la fundamentación de la propuesta de ley a discutir, debiendo explicitar la contribución que estas iniciativas en educación prestan al desarrollo del país.

De las dos fuentes mencionadas, recibe un trato preferencial la primera, por ser la más exhaustiva en términos de la política educativa; la otra se usa como complemento para iluminar, confirmar o contrastar los análisis que emergen de la primera. ${ }^{5}$ En todas ellas se realizó un análisis de los textos buscando el nexo entre la idea o visión de sociedad y la función que le cabe a la educación en dicho proyecto. Además, como complemento del análisis principal, se realizó un conteo del número de apelaciones explícitas a los conceptos principales de los distintos enfoques (énfasis democrático, énfasis económico y de desarrollo personal) en los discursos de inauguración del año escolar 1990-2009.

\section{RESULTADOS}

El análisis de la relación entre la educación y la visión sobre la sociedad chilena, en los discursos que justifican las políticas públicas en educación durante los años 1990 a 2009, permite distinguir tres etapas en el período. Ellas corresponden a las variaciones observadas en el modo de concebir la función de la educación en el orden social. Cambia aquello que se espera de la educación y los significados y desafíos que se le asignan. En lo que sigue, se indican los tópicos más relevantes para cada uno de estos momentos.

\subsection{PRIMERA ETAPA: 1990-1992}

Es posible distinguir un primer momento "fundacional" en el discurso político educativo de la Concertación. Este abarca los discursos de inauguración del año escolar de los años 1990 a $1992 .^{6}$ Sus características principales son las siguientes:

1. Se identifica con claridad la nueva etapa histórica caracterizada por la vuelta a la democracia y, en consecuencia, se transfieren las características y desafíos de la sociedad democrática a la política educativa. En el análisis cuantitativo se observa que, en este momento, las referencias a la democracia triplican a aquellas que se hacen a la dimensión de desarrollo económico. ${ }^{7}$

También se revisó los Mensajes Presidenciales del 21 de mayo de cada año, pero no entregaron aspectos substantivamente diferentes.

6 Los tres discursos son enunciados por Ricardo Lagos, ministro de educación del período en cuestión.

7 Las apelaciones a conceptos relativos a un enfoque democrático fueron un $65.7 \%$ del total, contra un $22.8 \%$ de las de tipo económico. 
Así, se postula que, en este nuevo orden, la educación debe estar construida por los distintos actores del país, quienes concurren a deliberar sobre los medios y los fines de la política educativa. Fundada en esta deliberación, la política educativa se propone como resultado del consenso y como política de un Estado encargado de implementar el mandato soberano del pueblo.

"Este es el sentido último de la democracia... Es a través del debate sobre las grandes metas y medios que se va formando el acuerdo sobre lo que querernos para la educación y la cultura. A las autoridades sólo les corresponde implementar ese consenso" (Discurso de Inauguración del año escolar [en adelante, D.I], 1990. R. Lagos).

Establecida esta perspectiva, se critica el sistema educativo heredado, tanto por su carácter mercantil, como por su inadecuación a las exigencias de contribuir al desarrollo personal, a la formación ciudadana y a la competitividad económica.

Respecto de la primera crítica, se señala:

"Desgraciadamente, una concepción errónea del rol del Estado ha llevado a maniatarlo en el cumplimiento de su obligación constitucional de garantizar el derecho a la educación para todos (...). El sistema nacional de educación, ha sido desarticulado. La responsabilidad social en educación ha sido remplazada por el ilusionismo del mercado. So pretexto de la necesaria descentralización, se ha llegado no sólo a la atomización del sistema, sino a una competencia a veces agresiva en donde debiera primar la cooperación" (D.I. 1990. R. Lagos).

Referido a la crítica sobre la funcionalidad educativa, se lee:

"en su conjunto, la educación chilena no satisface los requerimientos cualitativos de la sociedad y de las personas. No se logran en nuestras escuelas los aprendizajes que requiere y requerirá el desarrollo económico y científico-técnico del país. No se aprende en ellas como vivir en democracia. Tampoco se obtienen los valores, hábitos, habilidades y destrezas requeridas para el crecimiento personal o para enfrentar la vida cotidiana" (D.I. 1990. R. Lagos).

Estos textos ponen de manifiesto una concepción de sociedad democrática muy distinta a la heredada: dotada de un Estado fuerte y competente para actuar, que se haga responsable y garantice derechos y el desarrollo; articulada no sobre relaciones mercantiles individuales (atomización), sino sobre el crecimiento de las personas y la cooperación. La visión del Estado como activo y conductor del orden social, marca la diferencia con el período anterior de enfoque neoliberal, por lo que será recurrente en los discursos la alusión a este tipo de concepción estatal.

En orden al desafío democrático de igualdad, se resalta el déficit de equidad.

"Los beneficios de la educación se distribuyen insuficientemente y sin equidad. Las grandes mayorías reciben una educación de baja calidad (...), viven la ilusión de la escolaridad, a menudo descubriendo tardíamente que han recibido una formación irrelevante, que no les permite acceso al trabajo o al conocimiento superior, que no favorece la ciudadanía democrática ni ayuda a resolver sus problemas personales (...). Un sistema educacional de calidad para unos pocos es la negación del sistema democrático en el cual ahora queremos vivir" (D.I. 1990. R. Lagos).

Como norte se establece el derecho de todos a una educación de igual calidad, aspecto que debiera identificar la orientación de la política educativa del nuevo período democrático. Para lograrlo, al Estado le cabe un rol preponderante, dada 
"su obligación constitucional de garantizar el derecho a la educación para todos y no para una minoría, y en su tarea de fomentar eficazmente la calidad de la oferta educativa (...). La educación es un derecho de cada chileno o chilena. Por ello debe asumirse como una tarea nacional en la cual el Estado tiene una alta responsabilidad en cuanto representante de la sociedad. Esta educación debe basarse en el pluralismo y la libertad de enseñanza, la participación y responsabilidad de la comunidad y de los actores del sistema educacional" (D.I. 1990. R. Lagos).

La máxima expresión y exigencia del derecho y de una sociedad igualitaria se encuentra en la referencia a los más necesitados:

"Los pobres tienen derecho, no sólo a entrar a la escuela sino, sobre todo, a permanecer en ella y recibir una educación del mismo nivel de calidad que la de los más afortunados" (D.I. 1990. R. Lagos).

En cuanto a las orientaciones formativas que la educación debe proveer en el nuevo escenario social, se asienta la idea de una educación multifuncional, que habilite a las personas para actuar en las distintas y complejas dimensiones de la sociedad que se abre al siglo XXI.

"Una educación que ya no sólo forma ciudadanos o prepara para el trabajo como fueron las tareas de la educación del Siglo XIX y XX sino que además prepara a la gente para que pueda enfrentar la complejidad de la vida cotidiana y colectiva y ejercer el derecho a la felicidad" (D.I. 1990. R. Lagos).

2. Ya en 1991, el enfoque desde el cual se justifica la acción política comienza a transitar desde un énfasis democrático-fundacional, a una presencia creciente de un enfoque muy cercano al que propusieran, al año siguiente para América Latina, la CEPAL y la UNESCO, el cual instala la educación y el conocimiento como elementos centrales del desarrollo económico.

"Participamos de la convicción creciente que la clave del desarrollo de nuestros países en esta nueva etapa reside en incorporar a la economía conocimiento, tecnología avanzada y creatividad. Compartimos la necesidad que el desarrollo requiere una transformación productiva con equidad y cimentar el crecimiento en la incorporación del progreso técnico, que es esencial para el logro de competitividad, progreso que tiene como premisa fundamental un pertinente desarrollo educativo" (D.I. 1991. R. Lagos).

En el modelo de desarrollo productivo con equidad enmarcado en la sociedad del conocimiento, la educación pasa a ser un factor productivo clave.

"Una economía abierta y competitiva a nivel internacional significa en último término una capacidad para exportar valor agregado. La potencialidad que tenemos reside en la riqueza de nuestros productos primarios y en la incorporación del conocimiento humano. En último término, los países que progresan, son aquellos que exportan conocimiento y ello implica más y mejor educación" (D.I. 1991. R. Lagos).

Esta visión técnico-política, permite construir consensos entre las clases dirigentes relativas a la importancia de invertir en educación. Se verá en adelante, un predominio relativo de este modo de comprender la educación y, en consecuencia, una reducción de las indicaciones sobre las otras funciones educativas:

"Para que un Chile armado de conocimiento y dinamismo creativo, se inserte de modo apropiado en el mundo del futuro (...) la transformación (educativa) es, así vistas las cosas, 
un requerimiento estratégico que interesa no sólo a los educadores o a los especialistas" (D.I. 1991. R. Lagos).

La participación, elemento consustancial al orden democrático muy presente en 1990, se retoma en 1991, pero esta vez desde un marco más técnico-educativo relacionado con los procesos de enseñanza-aprendizaje:

"Queremos más responsabilidad, más participación de más gente, más discusión y también más consenso, depurado de toda consideración menor, de índole estrechamente partidaria o particularista (...) llamamos a la comunidad educacional a estudiar y a discutir las cuestiones propiamente educacionales. ¿Cómo se optimizan los procesos de enseñanza y aprendizaje? ¿Cómo se enriquece el currículum escolar con temáticas tan candentes y trascendentes como los derechos humanos?" (D.I. 1991. R. Lagos).

Asimismo, bajo el argumento técnico de eficiencia, se invita a la discusión sobre la descentralización, aconsejando debatir y estudiar nuevas y quizás mayores rectificaciones en este ámbito, toda vez que

“...conviene al desarrollo educacional profundizar y optimizar la descentralización, hacerla más participativa y eficiente y, de ningún modo, retroceder a formas superadas de centralización o burocratismo" (D.I. 1991. R. Lagos).

3. Por último, el discurso de 1992 retoma una concepción integral de la educación, visión que irá perdiendo presencia en los discursos posteriores. En él, junto al énfasis en el rol de la educación como eje del desarrollo -su función económica- se destaca también su función política y personal.

"Los chilenos nos hemos puesto de acuerdo en que la educación es fundamental para el desarrollo de la persona humana, para consolidar la democracia generando iguales oportunidades a todos y para convertirla en una palanca fundamental del progreso para poder participar en un mundo sin fronteras y en donde, en último término, el conocimiento y la técnica de sus habitantes son lo que nos permite construir un mundo mejor" (D.I. 1992. R. Lagos).

Sin embargo, la visión de sociedad propuesta por la coalición gobernante, se expresa en los objetivos de "integración social y desarrollo económico que la mayoría del país comparte” (D.I. 1992. R. Lagos). Esta visión subraya el componente económico del desarrollo, junto a un enfoque de integración al orden social y no tematiza explícitamente la democracia, en sus componentes de solidaridad, participación ciudadana y deliberación de los asuntos públicos y finalidades sociales, de forma que no existen metas declaradas a alcanzar por la educación en este ámbito.

Como síntesis de esta etapa, cabe destacar que los argumentos utilizados, relativos a los principios y justificaciones de la política educativa propuesta, tienen su base en el sistema democrático que se proyecta. Las funciones que de ella se esperan responden a una comprensión integral; vale decir, una visión que considera la educación para el desarrollo, entendido éste, como un avance en el aspecto económico: educación para la formación del capital humano; político: educación para el ejercicio ciudadano en democracia; desarrollo social: educación para la integración; y personal: educación para enfrentar la complejidad de la vida cotidiana en este siglo XXI. Es central en esta comprensión la referencia a la igualdad democrática, que debe expresarse en igualdad educativa. Estas demandas al sistema educativo desde un marco normativo que apela a la sociedad democrática, esto es, a su relación con la igualdad y a su importancia para la formación ciudadana, son fuertes al inicio, para mitigarse algo hacia el final de la etapa. 
Este segundo momento es el más largo: abarca trece años (1993-2005); se inicia al fin del primer gobierno de la coalición de partidos por la democracia, incluye el primer gobierno de seis años y casi la totalidad del segundo.

Lo extenso del período y la amplia acción político-educativa desplegada durante estos años, permiten afirmar que el discurso de esta etapa comunica las propiedades del proyecto político y educativo concertacionista. Mientras la primera etapa, por su carácter fundacional, buscó diferenciarse del orden político de la dictadura que la precede, esta segunda etapa expresa lo más propio del proyecto socioeducativo efectivamente llevado adelante por la Concertación. Se trata de la política de una coalición que, tras negociaciones, alianzas y la aplicación de estrategias de consensos, elabora un discurso que se distinguirá por los puntos presentados a continuación:

1. Los discursos de inauguración del año escolar de este sub-período -así como también los 'mensajes del ejecutivo'- consideran las tres funciones educativas; a saber, su importancia para el desarrollo personal, su cometido sociopolítico y su centralidad en el desarrollo económico.

Así, por ejemplo, se subraya:

"las grandes concordancias que el país ha generado en esta área: educación de calidad, educación que no discrimine socialmente, educación como base del desarrollo del país" (D.I. 1993. J. Arrate).

Otro texto claro, en este sentido, pertenece al mensaje de una de las leyes presentadas al Congreso:

"Hoy se quiere que nuestro país garantice una efectiva igualdad de oportunidades a todos los chilenos en materia educacional y laboral, mejore las condiciones de competitividad de la economía y establezca los fundamentos de un orden social inscrito en la modernidad y respetuoso de los valores, tradiciones, sentido de identidad y fortaleza de las instituciones democráticas" (Ley 18.876 de Reforma Constitucional que establece la obligatoriedad y gratuidad de la educación media. Mensaje presidencial, 2002).

Por último, un discurso indica:

"La democracia se la juega por la educación, porque es la llave del progreso del país y la herramienta fundamental para reducir la desigualdad (...), formar mejores ciudadanos (...), ser más solidarios; tolerantes y respetuosos del otro" (D.I. 2004. S. Bitar).

Los ejemplos aludidos -el primero al inicio del período, los otros dos casi al cierre del mismo- dan cuenta de la permanencia en la noción de una triple funcionalidad educativa. No obstante, vemos ya que el orden asignado a estas funciones expresa un predominio relativo del enfoque económico al explicitar el sentido de la política educativa.

2. De hecho, es posible constatar un relativo desplazamiento de la normatividad política democrática de la primera etapa, que -como vimos- subrayaba la participación ciudadana en la formulación de las políticas y una educación de igual calidad para todos,

El conteo de apelaciones muestra este desplazamiento: en este período los conceptos relacionados con la vinculación de la educación al desarrollo económico pasan a ser preponderantes: $45 \%$ de las apelaciones, contra un $37 \%$ relativas a la sociedad democrática. 
en aras de la importancia económica de la educación para el crecimiento económico, la inserción en los mercados globales y en la sociedad del conocimiento. ${ }^{8}$

Los textos en este sentido son múltiples y reiterativos:

"Como nunca antes, la suerte de los países depende del conocimiento y de la información, los que a su vez remiten a la educación (...) hoy la riqueza reside en la capacidad de las personas de usar el conocimiento para agregar valor, innovar y crecer. Estamos en la sociedad del conocimiento" (D.I. 1995. S. Molina).

"En la sociedad de la información, con la revolución en la tecnología de las comunicaciones, la educación vive profundas transformaciones a la vez que se instala como eje del tipo de sociedad que se quiere construir" (D.I. 1998. J.P. Arellano).

"En la década del 90 los chilenos trabajamos por un nuevo horizonte. Este horizonte ha puesto cada vez más sus urgencias y sus esperanzas en la educación, eje de esta sociedad del conocimiento" (...). "Hasta hoy, el conocimiento fue un bien escaso. Ya no lo es. La virtud relevante del futuro tiene que ver, entonces, con cómo usarlo y para qué usarlo (D.I. 2000. J.P. Arellano).

En este contexto, la educación deviene en factor clave al cual echar mano para participar en esta nueva sociedad y con ello alcanzar el estadio de desarrollo anhelado:

"Pero sólo la educación, la ciencia y la tecnología nos permitirán cruzar el umbral. Esa es nuestra apuesta estratégica. En el siglo XXI, en la sociedad del conocimiento, la gran competencia entre los países se da en la educación, la ciencia y la tecnología. Sólo aquellos que logren asegurar una educación de calidad, innovar y usar las nuevas tecnologías, podrán alcanzar el desarrollo y dar más igualdad. No hay un solo país en el mundo que haya alcanzado el progreso sin fortalecer y consolidar su educación. Por eso he afirmado que la educación es la riqueza de Chile"9 (D.I. 2004. S. Bitar).

3. La riqueza de la que se habla es el capital humano que debe aportar la educación, pues este capital pasa a ser condición de una economía fundada en la agregación de valor a partir del conocimiento.

La formación de capital humano es el referente societal que justifica la modernización del sistema educativo que se busca; en ese marco adquiere inusitada centralidad la calidad de la educación media, medida según resultados académicos o el rendimiento escolar. Algunos discursos de inauguración del año académico son elocuentes:

"Hoy todos sostenemos que la modernización de la educación es la clave de nuestro porvenir. La tarea de Chile es, entonces, multiplicar la eficiencia de los medios humanos y materiales (...), concretar las grandes concordancias que el país ha generado en esta área: educación de calidad, que no discrimine socialmente, educación como base del desarrollo del país" (D.I. 1993. J. Arrate).

"Si nuestro objetivo es mejorar los aprendizajes de nuestros estudiantes, tenemos que poner más atención en los resultados escolares (...). Una educación de calidad para cada niño es un derecho y una responsabilidad insoslayable (...); contamos con los recursos educativos en nuestras escuelas y liceos para centrar nuestros esfuerzos en el rendimiento escolar, en los resultados" (D.I. 2002. M. Aylwin).

9 "La educación es la riqueza de Chile" corresponde al eslogan representativo de la política educativa en el gobierno de Ricardo Lagos (2000-2005). 
En línea con esta voluntad de centrar los esfuerzos en la obtención de resultados académicos, el discurso político-educativo comenzará a referirse a los medios para alcanzarlos. Así, y en forma creciente, el discurso político incorpora aspectos técnico-pedagógicos que se consideran importantes para los resultados académicos, como la reforma curricular, la Jornada Escolar Completa, la política docente. Cabe recordar que todas estas estrategias, ya sea de diseño institucional o propiamente pedagógicas, son medios para alcanzar la calidad educativa, que se concibe como la principal meta u objetivo del sistema.

4. La función de la educación para la formación personal emerge también como componente de la calidad educativa, a través de la reforma curricular iniciada en esta etapa.

Si bien es una función enunciada ya en el primer discurso de 1990, es en esta fase, en especial durante la segunda mitad de la década del ' 90 , en correspondencia con el cambio curricular, cuando se le da contenido, en tanto se despliegan los atributos en torno a los cuales debiera formarse a las personas. ${ }^{10}$ En sus planes y programas, y en sus objetivos, el curriculum deberá promover valores como la autonomía, la creatividad, la responsabilidad y la libertad. Esta demanda responde tanto a objetivos de funcionalidad económica: creatividad y libertad para participar en los mercados, como de funcionalidad política, para el desarrollo de una convivencia social que integre a partir de la responsabilidad, el respeto, la tolerancia.

El discurso de inauguración de 1997 señala:

"El proceso global de modernización está transformando profundamente la vida en la sociedad contemporánea (...), estos cambios afectan fuertemente al mundo de la producción y transforman las organizaciones y la vida cotidiana de todos (...). Para el mundo que viene una auténtica educación debiera fomentar y nutrir las capacidades de ser y actuar libremente y, al mismo tiempo, respetar los ordenamientos que hacen posible una vida ciudadana que beneficia a todos, acrecentando las capacidades de percibir y vivir en el respeto a derechos $y$ deberes (...). Debiera especialmente, y en particular en un país como el nuestro, todavía herido por la pobreza de muchos, ser capaz de sensibilizar y convocar a la construcción de una sociedad más equitativa y solidaria” (D.I. 1997. J.P. Arellano).

En otro discurso se anota:

"Aprender más y mejor implica no sólo una adecuada formación en el ámbito de los conocimientos, sino también en el desarrollo personal, en la formación de valores, actitudes, habilidades y destrezas que les permitan aprender a convivir, a discernir, a ser capaces de enfrentar los desafios del mundo de hoy" (D.I.2001. M. Aylwin).

5. Llegados hasta aquí caben dos observaciones sobre la centralidad que ocupa en el discurso, la idea de calidad educativa.

En primer lugar, pese a la amplitud del concepto de calidad educativa este se asocia principalmente al mejoramiento de los aprendizajes cognitivos medido a través de resultados académicos, siendo la prueba SIMCE -y su importancia creciente al interior del sistemael mejor ejemplo en este sentido; son menos enfatizadas otras dimensiones de la calidad más próximas a la formación ciudadana, a la formación moral y al desarrollo personal.

10 En el conteo de apelaciones, si bien la referencia al desarrollo personal es siempre minoritaria con relación a las otras dimensiones, es en esta etapa cuando logra más peso relativo: un 19\% de las apelaciones, contra $11.4 \%$ en el primer período y $13.9 \%$ en el tercero. 
En segundo lugar, como es sabido, el discurso educativo de la Concertación se levantó en torno a dos ejes: calidad y equidad. ${ }^{11}$ Mientras la calidad educativa aparece comúnmente asociada a los objetivos de desarrollo, competitividad y crecimiento, la equidad emerge mucho más próxima al campo de la igualdad y la democracia. Sin embargo, ambos criterios no reciben un trato equivalente en el discurso y la calidad educativa adquiere una creciente importancia tanto en términos formales (presencia de cada categoría en el discurso), ${ }^{12}$ como en la mayor importancia o valoración que adquiere el tema de la calidad, quedando la equidad sin criterios de exigencia y como una de las consecuencias de la calidad. Este trato asimétrico de ambos criterios tiene como correlato el modo como el discurso vincula el rol de la educación al orden social: la preponderancia del tema de la calidad, expresa un predominio del enfoque económico en la narrativa de política educativa. Una cita expresiva:

"De la calidad de nuestra educación depende: la más dura de nuestras exigencias: un crecimiento sostenido y sustentable; el más preciado de nuestros valores: la equidad social; superar nuestro mayor dolor: la pobreza; fortalecer nuestra principal misión: la convivencia democrática" (D.I. 1998. J.P. Arellano).

6. Lo dicho hasta acá no significa que la exigencia democrática haya desaparecido del discurso de política educativa; ella se hace presente principalmente en torno a las ideas de equidad e igualdad de oportunidades. En este sentido, las políticas educativas con fines para el fortalecimiento democrático serán aquellas orientadas bajo el criterio de la equidad y evaluadas según su contribución a la igualdad de oportunidades -o más precisamente, a la reducción de la pobreza. ${ }^{13}$ En este marco, la estrategia de focalización de los recursos fiscales y las políticas de discriminación positiva se levantan como instrumentos idóneos a estos objetivos.

"Mejorar la educación quiere decir hacerla accesible para todos, garantizando un nivel adecuado. Por eso hemos impulsado políticas destinadas a fortalecer la educación en las zonas y en los grupos sociales más desfavorecidos" (D.I. 1993. J. Arrate).

"También la educación es el factor principal para superar la marginalidad y la pobreza y lograr una mayor igualdad social. Es quizá la única que permite alcanzar cambios de un carácter más permanente en las condiciones individuales y sociales, otorgando las posibilidades para que cada persona, por sus propios medios, pueda acceder a mejores y mayores oportunidades de desarrollo personal, social, económico y cultural" (D.I. 1996. S. Molina).

"En contextos de pobreza, donde las familias tienen menores recursos educativos y difíciles condiciones económicas (...) es un imperativo de justicia tener una política de discriminación positiva que permita lograr una efectiva igualdad de oportunidades” (D.I.2001. M. Aylwin).

"Tenemos el compromiso de fortalecer la educación pública, porque es ella la que acoge a los sectores más pobres, a quienes el Estado tiene el deber de privilegiar. Si la educación pública no logra dar una atención de calidad a los alumnos de menores recursos, no cumplirá con su rol y el país no alcanzará ni desarrollo ni justicia" (D.I. 2005. S. Bitar).

11 Una muestra clara de esto fue el discurso con que se instaló el Programa de las 900 escuelas, primera iniciativa educativa de la Concertación y el nombre de los programas MECE (básica en 1991 y media en 1995), sigla que significa: "Mejoramiento de la equidad y de la calidad de la educación". Para una revisión exhaustiva de las políticas educativas implementadas por los primeros gobiernos de la Concertación, ver Cox (2003).

12 Las apelaciones a conceptos relativos a un enfoque de calidad fueron cuatro veces más que las referidas a equidad, contra un $22.8 \%$ de las de tipo económico.

13 Este objetivo es central, pues en materia de distribución, los gobiernos de la Concertación utilizaron las políticas sociales como principal mecanismo distributivo, al margen del mercado; esto es, sin intervenir en la distribución de los factores productivos de ingreso (Vega, 2007). 
"Mejor educación genera más democracia. Por eso la prioridad de los gobiernos democráticos es la educación. La educación incide en la persona y su familia, tiene un impacto en la justicia social, genera más igualdad de oportunidades y hace de Chile una nación más pujante, justa y libre” (D.I. 2005. S. Bitar).

Esta visión sobre la equidad en educación, muy centrada en la igualdad de oportunidades, no aparece muy exigente de cara a una visión de democracia que postule el reparto de lo común conforme a la categoría de ciudadano, entendido como sujeto de iguales derechos. Más bien, las indicaciones democráticas se expresan teñidas por una comprensión económica del bienestar social e individual, aspirando a igualar las condiciones de vida, a evitar la marginalización social y reducir la pobreza. Una cita ejemplar al respecto es enunciada por el Ministro Bitar:

"Si ustedes -niños, niñas y jóvenes- tienen mejor educación, lograrán mejores empleos y tendrán mejores sueldos” (D.I. 2004. S. Bitar).

Esta consideración relativa de la igualdad permite señalar que el discurso define mucho más una idea de igualdad educativa compatible con una educación de mercado, donde el bien común asegurado a todos es un mínimo o base común, por sobre el cual se admite las diferencias.

En resumen, en esta fase, predomina una normatividad democrática pensada como igualdad de oportunidades y focalización de los recursos en los más pobres, mientras que temáticas igual o más consustanciales a este orden, como son la participación ciudadana, la cohesión social y el ideal de igualdad, se tematizan más parcialmente.

A partir de las indicaciones señaladas, sostenemos que la segunda etapa del discurso se diferencia por una perspectiva caracterizada por: i) la función social de la educación pierde complejidad y poder, al punto que el discurso adolece de un sesgo economicista, subrayando principalmente su contribución para la formación del capital humano del país; en contrapartida, se reduce su contribución a la política. ii) Dicha funcionalización educativa tiene su correlato en una determinada mirada sobre la sociedad. La democracia como marco teórico-práctico para justificar la acción del Estado en educación no tiene suficiente peso y predomina una lectura del enfoque cepalino de transformación productiva con equidad, caracterizada, entre otros, por la adaptación a un orden global de mercado y a la tecnocratización de los asuntos de la vida pública.

\subsection{TERCERA ETAPA: 2006-2009}

La tercera y última etapa, retoma todos los elementos en torno a los que se ha constituido el discurso político educativo de la Concertación y que presentamos recién. Continúa el enfoque de capital humano en la comprensión sobre los fines sociales de la educación y la calidad educativa permanece como la principal meta del sistema. Sin embargo, esta orientación de continuidad se complementa con una vuelta al énfasis especial en los argumentos relativos a un horizonte normativo democrático y de justicia social. Es justamente la reutilización en el discurso de este enfoque -distintivo de la primera etapa- lo que nos permite diferenciar este último momento del precedente. ${ }^{14}$

14 El conteo de apelaciones muestra que hay una fuerte recuperación del discurso democrático, pero que su preponderancia sobre el discurso económico no es tan fuerte como en la primera etapa. Las apelaciones a la democracia llegan al 50\% y a la importancia de la educación para el desarrollo económico a un $36.1 \%$ de las menciones (en la primera etapa eran $65.7 \%$ y $22.8 \%$ respectivamente). 
1. El 2006 se inicia con un discurso que reconoce el predominio que ha tenido el enfoque económico en la definición de los fines educativos, por sobre una mirada político democrática. No se considera el énfasis económico como una opción errada de la política educativa, pero se advierte que es una mirada limitada e insuficiente de cara a las exigencias que la democracia hace a la educación.

"Nos hemos habituado - iy no es malo que así haya ocurrido! - a pensar la educación, primero que todo, como un motor de la economía, de nuestra competitividad en los mercados internacionales y de nuestra prosperidad interna. Este se refleja incluso en el lenguaje que usamos para hablar de la educación: hablamos de ella como un factor de producción, como nuestro capital humano, como un elemento clave para mejorar la productividad del trabajo. ¡Y así es!” (D.I. 2006. M. Hornkhol).

Sin embargo, se admite que la educación:

“No sólo es (...) condición para el crecimiento económico sino tan, o tanto más importante, la educación es ahora también la base sobre la cual constituimos nuestra identidad como nación, construimos nuestras vidas, donde se asientan nuestras libertades y el punto de entrada hacia una mayor igualdad y justicia sociales (...). Tiene que ver con cómo mujeres y hombres se hacen cargo de sus vidas; afirman y defiende sus derechos; asumen responsabilidades y se integran activamente en la comunidad" (D.I. 2006. M. Hornkhol).

Observamos un discurso político que avanza en reflexividad, pues tematiza su propio desarrollo -reconociéndolo como limitado- para incorporar principios normativos democráticos y de autonomía personal, los cuales, de ser incluidos, harían posible justificar otras propuestas y finalidades del orden social.

2. Al discurso de 2007 lo precede el movimiento secundario de 2006 que denuncia un sistema educativo reproductor de las desigualdades sociales. ${ }^{15}$

Con ello, se instala el tema de la desigualdad en el centro de la reflexión de política educativa y en el discurso vuelven a tener mayor cabida exigencias provenientes de un ideal normativo democrático. Así, se alude a la participación, responsabilidad y soberanía ciudadana para decidir y evaluar los asuntos comunes en orden a construir una sociedad más justa e igualitaria. A su vez, se valora la educación en tanto derecho ciudadano y por tanto, la presencia de un Estado activo, comprometido en garantizarlo, y en promover instituciones fundadas en un principio de justicia comúnmente acordado.

"En esta etapa, las políticas se construyen en diálogo con las demandas sociales. Hemos estado y lo seguiremos haciendo, dialogando con los actores educativos y trabajando en conjunto la solución de los problemas" (D.I. 2007. Y. Provoste).

Junto con proponer la participación, se crítica a la desigualdad:

"Quiero insistir: la desigualdad no es una condena con la que tenemos que vivir como país, es un mandato ético a trabajar cada día, para que todos puedan alcanzar sus sueños de vivir de una manera mejor. La educación es uno de los derechos fundamentales de los ciudadanos y garantizarla como un servicio de calidad pasa a ser una nueva y central tarea del Estado de nuestro gobierno" (D.I. 2007. Y. Provoste).

15 Las dos grandes reivindicaciones del movimiento "pingüino" fueron el fortalecimiento de la educación pública y el rechazo al lucro en educación (o sea a la educación de mercado). Sobre el movimiento estudiantil ver García-Huidobro (2007). 
"La ciudadanía exige mayor presencia del Estado en la educación (...). Por ello ha llegado el momento de abordar decididamente los problemas institucionales que afectan la calidad y equidad de nuestro sistema educativo" (D.I. 2007. Y. Provoste).

3. El enfoque democrático y las exigencias que reclama, están presentes también en el discurso de inauguración del año escolar de 2008.

La igualdad como normatividad orientadora de la política educativa, el rol activo del Estado y la reforma a la institucionalidad educativa, son las temáticas que ordenan el discurso.

"Si para nosotros uno de los grandes objetivos como país es avanzar hacia un país más igualitario, donde cada uno de nuestros hijos e hijas, independiente de la cuna donde nació o el lugar del país donde nació, tengan todas las oportunidades que se merecen, la educación sin duda está en el centro de esas políticas (...). "Jóvenes que han tenido distintas calidades en su educación (...) compiten con la cancha dispareja. Algunos parten de más arriba y otros parten de más abajo. Por lo tanto, equidad, igualdad de oportunidades, significa no tratar a todos por igual, porque la gente parte de distintos puntos de partida y, por tanto, tiene que darse más a quienes tienen más dificultades" (D.I. 2008. Y. Provoste).

Dos condiciones se sostienen como requisito para la realización de políticas educativas que promuevan la igualdad.

La primera, siempre presente en el discurso, refiere a la importancia del rol del Estado: "El Estado tiene una responsabilidad fundamental en la educación. Sin su presencia, simplemente no hay educación para quienes más lo necesitan. Sin su acción, no hay posibilidad de avanzar hacia una educación de igual calidad para todos (...); necesitamos un Estado que modernice y perfeccione su acción, no un Estado que retroceda" (D.I. 2008. Y. Provoste).

La segunda es el cuestionamiento a la actual organización del sistema educativo. Esta crítica estaba también presente en el primer discurso del año 1990, a objeto de indicar su carácter antidemocrático y distinguir, de esta forma, el nuevo período democrático que comenzaba. Sin embargo, a poco andar -ya en 1991- la crítica a la institucionalidad desaparece de la narrativa política educativa; sólo ahora se retoma, a fin de indicar críticamente sus consecuencias negativas para el alcance de un orden justo e igual. "Estamos en medio de un profundo proceso de reforma, que busca modificar la estructura de nuestro sistema educativo" (D.I. 2008. Y. Provoste).

4. Por último, en el discurso de 2009 está menos presente el uso de las ideas democráticas de participación e igualdad para argumentar la racionalidad de las medidas educativas, pese a lo cual el listado de acciones a realizarse termina por referirlas al ideal de sociedad democrática: "Son estas tareas, las que se encuentran a la base de una sociedad democrática, más equitativa, inclusiva y más justa” (D.I. 2009. M. Jiménez).

En suma, en esta tercera etapa se advierte un discurso de continuidad con la concepción de educación como capital humano, presente desde la primera etapa y preponderante en la segunda. Pero, al mismo tiempo, hay apelaciones que apuntan al ideal de igualdad como principio normativo-ético y como referente para la elaboración de las políticas y el diseño de una nueva institucionalidad para la educación. En este contexto, extraña que la promoción y formación para la participación por medio de una educación para la ciudadanía no aparece destacada. 


\section{CONCLUSIONES}

Para cerrar el recorrido por el discurso de política educativa de los veinte años de gobiernos de la Concertación, retomaremos sucintamente los tres momentos analizados y nos detendremos enseguida en la orientación permanente, que cruza las tres etapas.

Como vimos, el primer momento es el lapso de la transición, en el que se marca con claridad la identidad del nuevo gobierno democrático. Es un discurso más rico y complejo que el de las etapas posteriores. Vincula la educación al crecimiento económico y al desarrollo, pero también quiere marcar diferencias con la sociedad heredada de la dictadura.

Lo primero se expresa en la lógica de la reflexión sobre los cambios productivos introducidos por la sociedad de la inteligencia, que suponen una incorporación del conocimiento a la producción y ponen, por tanto, en un lugar de importancia a la educación.

Lo segundo puede sintetizarse en el acento en dos exigencias democráticas: participación e igualdad. La voluntad de construir la sociedad a través de la participación ciudadana, se enuncia en la idea de generar una política educativa consensuada, pero fruto de un amplio debate. La igualdad educativa se postula de modo contundente mediante la exigencia de una educación de la misma calidad para ricos y pobres.

La segunda etapa estuvo marcada por la acción, las reformas y realizaciones. En este período se continúa y adquiere gran substantividad en el discurso político-educativo la necesidad de una educación que aporte al crecimiento económico. Es en torno a esta orientación que se busca un consenso amplio en materia educativa, que permite el juego de continuidad y cambio característico de la política de la Concertación en educación: mantención del modelo y políticas de ampliación y mejoramiento de la calidad y equidad.

En el período de cierre, el Gobierno enfrenta demandas educativas fuertes, porque cuentan con amplio apoyo ciudadano y por su radicalidad: se demanda un cambio de modelo. En ese contexto, el discurso reencuentra las características más democráticas del discurso inicial: política educativa que se elabore en diálogo con la ciudadanía y recuperación de la exigencia de educación más igualitaria. Pero ya no hay tiempo para modificar la acción. Además, hay que observar que, en este retorno, el discurso del 2006-2009 no llega a criticar la mercantilización de la educación ni a poner al centro su responsabilidad de enseñar a vivir en democracia, contenidos presentes en el discurso del 1990-1992.

Como constante, en medio de estas variaciones, el contenido del discurso de política educativa de los gobiernos de la Concertación -sus objetivos, metas, y atributos contribuyentes a la estrategia de desarrollo social- expresa una orientación normativa, central que puede calificarse como económico-desarrollista y que sirve de base a la justificación de las acciones emprendidas. En ella, el crecimiento económico se concibe como factor esencial y prioritario en el avance al desarrollo y, en consecuencia, la primera tarea del sistema educativo consistirá en responder a los requerimientos de capital humano de una economía con mercados libres, abierta y globalizada.

En esta narrativa, el objetivo central de la política educativa es la 'calidad educativa', identificada con resultados académicos. La equidad termina siendo un objetivo segundo y consecuencia del primero, como resultado de los avances en calidad, mejorarían las oportunidades de las mayorías $\mathrm{y}$, de este modo, la educación sería una contribución a una mayor igualdad de oportunidades.

Dada esta orientación central, el discurso se ve muchas veces dominado por un enfoque economicista (o productivista) para observar los fines educativos. La mirada educativa 
se reduce a su función instruccional para la formación del capital humano, la que, a su vez, identifica resultados educativos con los que pueden ser medidos. Con esto quedan en segundo plano y con una pérdida relativa de peso en el discurso los objetivos políticos, esto es las exigencias que el desarrollo de la democracia debe hacerle a la educación y que fueron parte del ideario democrático fundacional de la Concertación.

La indicación anterior no implica deducir ausencia u omisión de la función educativa en relación a un orden normativo democrático; en efecto, los elementos de igualdad y justicia aparecen, tanto en el momento inicial fundante y, en buena medida también, al final del período Concertacionista. Con todo, este discurso porta un déficit en nociones propiamente democráticas como son la igualdad, la participación y la cohesión social; se ven, así, debilitadas las representaciones simbólicas que sustentan la democracia como modelo orientador del orden social. Correlativamente, puede observarse que la función educativa se despolitiza, pues se la representa menos como herramienta para formar ciudadanos interesados en un mejor orden social. La utilización parcial del ideal democrático -que destaca la igualdad de oportunidades, dejando de lado otros elementos sustantivos como la justicia, la participación o la cohesión social- devela una debilidad comprehensiva en la concepción de este ideario u horizonte normativo para la orientación de la política educativa.

\section{REFERENCIAS BIBLIOGRÁFICAS}

\section{Fuentes primarias}

Discurso del Ministro de Educación Señor Ricardo Lagos Escobar en el Instituto Nacional. 22 de Marzo de 1990.

Discurso del Ministro de Educación, Señor Ricardo Lagos, en la ceremonia de inauguración del año escolar. Concepción, 5 de Marzo de 1991.

Discurso del Ministro de Educación, Señor Ricardo Lagos Escobar, en la inauguración del año escolar. Antofagasta, 9 de Marzo, 1992.

Discurso del Ministro de Educación, Señor Jorge Arrate Mac Niven. Escuela Villa Las Estrellas Territorio Antártico chileno, 8 de marzo de 1993.

Discurso del Ministro de Educación, Señor Sergio Molina, en inauguración año escolar. Liceo Juan Gómez Milla, Santiago, 06 de marzo de 1995.

Discurso del Ministro de Educación, Señor Sergio Molina S, en la inauguración del año escolar. Santiago, Liceo A-70 Amador Neghme Rodríguez, Estación Central, Marzo de 1996.

Discurso del Ministro de Educación, Señor José Pablo Arellano, en la inauguración del año escolar. Copiapó. 1997.

Discurso del Ministro de Educación, Señor José Pablo Arellano, en la inauguración del año escolar. Escuela Los Jazmines, Melipilla, 6 de marzo de 1998.

Discurso del Ministro de Educación, Señor José Pablo Arellano, en la inauguración del año escolar. Escuela Los Mandarinos, Peñaflor, 1999.

Discurso del Ministro de Educación, Señor José Pablo Arellano, en la inauguración del año escolar. Escuela Manuel Rojas, Macul, marzo, 2000.

Discurso de la Ministra de Educación, Mariana Aylwin O. en la Inauguración del Año Escolar. Escuela 583, El Bosque, marzo 2001.

Discurso de la Ministra de Educación, Mariana Aylwin O. en la Inauguración del Año Escolar. Liceo Andrés Bello de Puerto Montt, 5 de marzo de 2002. 
Discurso del Ministro de Educación, Sergio Bitar, en la Inauguración del Año Escolar, en la localidad de Diego Almagro, en la Región de Atacama, 2003.

Discurso del Ministro de Educación, Sergio Bitar, en la Inauguración del Año Escolar, en la localidad de Diego de Almagro, 2004.

Discurso del Ministro de Educación, Sergio Bitar, en la Inauguración del Año Escolar, Centro Educacional Padre Alberto Hurtado, San Bernardo, 2005.

Discurso de la Ministra de Educación, Marigen Hornkohl, en la Inauguración del Año Escolar. Escuela Víctor Jara de Santiago, 2006.

Discurso de la Ministra de Educación, Yasna Provoste, en la Inauguración del Año Escolar. Colegio Santa Irene, La Florida, 12 de marzo, 2007.

Intervención de S.E. la Presidenta de la República, Michelle Bachelet al inaugurar año escolar. Valparaíso, 5 de marzo de 2008.

Discurso de la Ministra de Educación, Mónica Jiménez, en la Inauguración del Año Escolar. Escuela Senderos del Saber, Comuna de San Ramón, Santiago, 2 de Marzo de 2009.

Ley 19.070 de estatuto docente. Mensaje presidencial. 1990.

Ley $\mathrm{n}^{\circ}$ 19.284. Establece normas para la plena integración social de personas con discapacidad. 1994.

Ley $\mathrm{n}^{\circ}$ 19.410. Introduce modificaciones a la ley $\mathrm{n}^{\circ} 19.070$ y DFL $\mathrm{n}^{\circ} 5$ de educación de 1993, y establece los beneficios que indica. 1995.

Ley 19.532 de jornada escolar completa. Mensaje presidencial. 1996.

Ley 18.876 de reforma constitucional que establece la obligatoriedad y gratuidad de la educación media. Mensaje presidencial. 2002.

Ley 20.219 que establece un sistema nacional de aseguramiento de la calidad de la educación superior. Mensaje presidencial. 2003.

Ley $N^{\circ} 19.873$. Crea Subvención Educacional Pro- Retención de alumnos y establece otras normas relativas a las remuneraciones de los profesionales de la Educación, 2003.

Ley 19.961 de evaluación docente. Mensaje presidencial. 2004.

Ley 20.248 de subvención escolar preferencial. Mensaje presidencial. 18.10.2005.

DTO-196. Reglamento sobre obligatoriedad de establecimientos educacionales de contar con a lo menos un $15 \%$ de alumnos en condiciones de vulnerabilidad socioeconómica como requisito para impetrar la subvención. 2005.

Proyecto de reforma constitucional que establece como deber del Estado velar por la calidad de la educación. 2006.

DFL $\mathrm{N}^{\circ}$ 2. Ley general de educación. Mensaje presidencial. 2007.

Ley $\mathrm{N}^{\circ}$ 20.162. Modifica el numeral 10 del artículo 19 de la Carta Fundamental, con el objeto de establecer la obligatoriedad y gratuidad del segundo nivel de transición de la educación parvularia. 2007.

Proyecto de ley que crea una Superintendencia de Educación. 2007

\section{Fuentes secundarias}

Arendt, H. (2005). La condición humana. Barcelona: Paidós.

Becker, G., (1983). El capital humano. Madrid: Alianza Editorial.

Castells, M. (2005). Globalización, desarrollo y democracia. Chile en el contexto mundial. Santiago de Chile: FCE.

CEPAL (1990). Transformación productiva con equidad. La tarea prioritaria del desarrollo de América Latina y el Caribe en los años noventa. Santiago de Chile: Naciones Unidas - CEPAL.

CEPAL-UNESCO. (1992). Educación y Conocimiento, Eje de la Transformación Productiva con Equidad. Disponible en: http://www.egeneros.org.mx/admin/archivos/lec_equida.pdf

Cortina, A. (1997). Ciudadanos del Mundo. Hacia una Teoría de la Ciudadanía. Madrid: Alianza Editorial. 
Cox, C. 2003. Políticas educacionales en el cambio de siglo: la reforma del sistema escolar de Chile. Santiago de Chile: Ed. Universitaria.

Dubet, F. (2007). "El declive y las mutaciones de la institución". Revista de Antropología Social, n.16, 39-66. . (2002). El declive de la institución. Barcelona: Gedisa.

Durkheim, E. (1998). Educación y pedagogía. Buenos Aires: Editorial Losada. . (1977). La educación moral. Buenos Aires: Editorial Losada.

García-Huidobro, J.E. (2007). “¿Qué nos dicen las movilizaciones estudiantiles del 2006 de la visión de los estudiantes sobre la educación secundaria?” En Dussell, I. et al., III Foro Latinoamericano de Educación: Jóvenes y docentes en el mundo de hoy (pp. 87-110). Buenos Aires: Santillana.

Guttman, A. (2001). La Educación Democrática. Una Teoría Política de la Educación. Buenos Aires: Paidos.

Habermas, J. (1989). Teoría de la acción comunicativa. Complementos y estudios previos. Madrid, Cátedra.

Peña, C. (2008). "El Concepto de Cohesión Social, Debates teóricos y usos políticos". En Tironi, E. (ed.), Redes, Estado y Mercado, Soportes de la cohesión social latinoamericana (pp. 2952). Santiago: Uqbar.

Rancière, J. (2006). Política, policía, democracia. Santiago de Chile: LOM.

Tedesco, J. C. (1998). Reformas educativas en América Latina: Discusiones sobre equidad, Mercado y políticas públicas. Talca: Universidad de Talca. (1995). El Nuevo Pacto Educativo. Madrid: Anaya.

Tironi, E. (2005). El sueño chileno, Comunidad, familia y nación en el Bicentenario. $3^{\mathrm{a}}$ ed. Santiago: Taurus.

UNESCO (1996). La educación encierra un tesoro, Informe de Comisión internacional sobre la educación del siglo XXI. Presidida por Jacques Delors. Madrid: Santillana.

Vega, H. (2007). En vez de la injusticia: un camino para el desarrollo de Chile del siglo XXI. $2^{\mathrm{a}}$ ed. Santiago: Debate. 
